\title{
Protocol Development for 3D Reconstructions: Combining In Vivo Imaging, APEX2 and En Bloc Staining of Mouse Visual Cortex.
}

\author{
JoAnn Buchanan ${ }^{1}$, Marc Takeno ${ }^{1}$, Tanya Daigle ${ }^{1}$, Adam Bleckert ${ }^{1}$ Daniel Bumbarger ${ }^{1}$, Agnes Bodor ${ }^{1}$, \\ Derrick Brittain $^{1}$, Ali Cetin ${ }^{1}$ and Nuno Maçarico da Costa ${ }^{1}$. \\ 1. Allen Institute for Brain Science, Seattle, WA, USA.
}

The emerging discipline of connectomics has fueled interest in the development of methods for high throughput 3D electron microscopy methods for large volumes of brain. These methods require tissue processing methods that produce sharply delineated membranes with sufficient contrast to identify them in consecutive serial sections and allow manual and automated segmentation of the data. In order to facilitate this reconstruction and aid the identification of different cell types, we are using the genetically encoded EM markers APEX and APEX2 [1,2], to trace individual neurons, in combination with deep penetration en bloc staining to allow reconstruction of entire circuits. These aims are directed at reaching our final goal of combining multiphoton in vivo imaging of $\mathrm{Ca}^{2+}$ activity from the cortex of an awake mouse, followed by large-scale serial sectioning transmission electron microscopy (ssTEM) reconstruction of $1 \mathrm{~mm}^{3}$ from the same imaged area.

To achieve this goal, we will begin with a sparse reconstruction of APEX2 [1] labeled neurons. We have expressed this engineered peroxidase label in vivo in a Cre-dependent manner to allow correlated LM and EM. Following in vivo imaging, the tissue is fixed and the APEX2 reacted with DAB and $\mathrm{H}_{2} \mathrm{O}_{2}$ to produce an electron-dense precipitate that can be seen in vibratome sections. The sections are processed for EM and $40 \mathrm{~nm}$ serial sections cut and imaged. The APEX2 label facilitates serial section image registration and alignment. Figure 1 shows correlative imaging of virus-mediated direct expression of APEX peroxidase. Figure 2 shows virus-mediated recombinant APEX2 peroxidase expression in a transgenic (Rorb-IRES2-Cre) Cre-dependent mouse line.

Our long-term goal is to prepare EM samples for a large-scale, dense reconstruction of a block of the mouse visual cortex, where activity was previously recorded by multiphoton $\mathrm{Ca}^{2+}$ imaging in an awake, behaving mouse. This requires tissue with high en bloc contrast and tissue preservation throughout the depth of the block. We are testing protocols that include optimizing reduced osmium (RO) and osmium bridging (OTO) for contrast enhancement with techniques to improve penetration in brain samples up to $1.5 \mathrm{~mm}^{3}$. In our hands, the reduced osmium OTO (osmium ferricyanide-thiocarbohydrazide-osmium ferricyanide) provides good contrast, but leads to uneven penetration and infiltration problems. To circumvent these issues, we are examining resin formulations suggested by a previous study of whole mouse brain [3] as well as implementing both ultrasonic and microwave assisted osmium staining. Because we will cut up to 30,000 ultrathin serial sections, it is critical that sections have adequate contrast and that resin be uniformly polymerized throughout to facilitate serial sectioning.

[1] S. Lam et al., Nature Methods, 12 (2015) p. 51.

[2] J. Martell et al., Nature Biotechnology, 30 (2012) p. 1243.

[3] S. Mikula, J. Binding, and W. Denk. Nature Methods, 9 (2012) p. 1198. 
[4] The authors are grateful to the Allen Institute founders, P.G. Allen and J. Allen, for their vision, encouragement, and support.
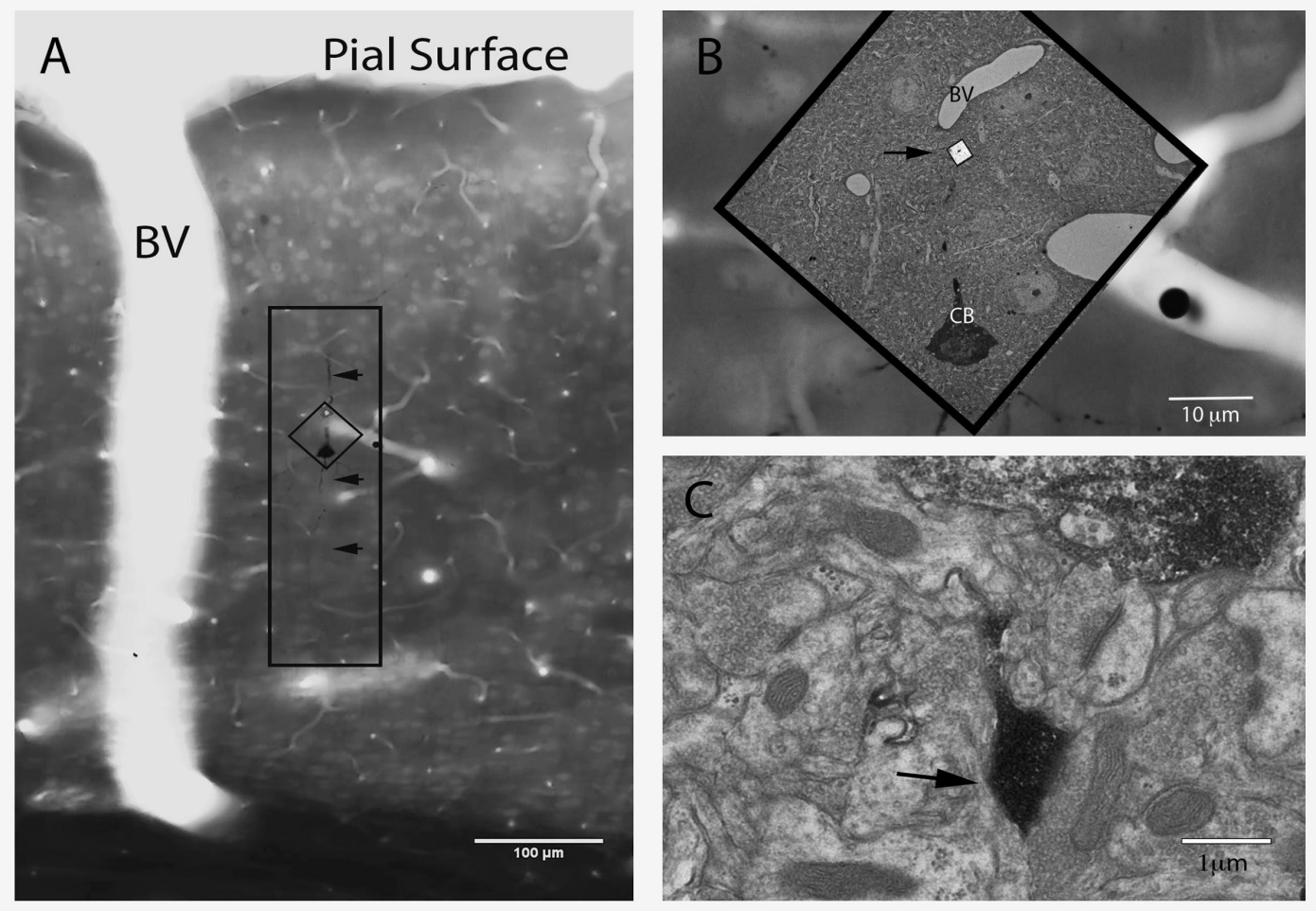

Figure 1. APEX labelled neuron in layer 4 of mouse visual cortex. (A) Rectangle outlines the neuron and APEX reaction product filing the cell (arrows) in a $60 \mu \mathrm{m}$ vibratome resin embedded slice by LM. Diamond boxed area shown in panel B. Blood vessel and pial surface of the brain are evident. (B) Electron micrograph showing the cell body (CB) and apical dendrite, filled with electron-dense reaction product. Arrow points to boxed area shown in C. (C) High magnification shows APEX labelled dendrite (arrow) making a synapse.
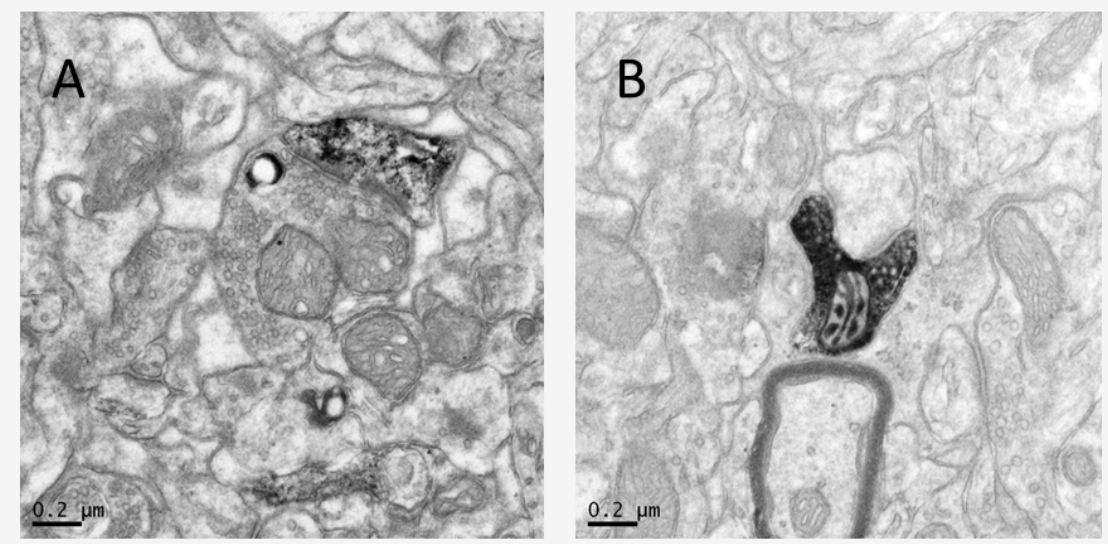

Figure 2. Cre-dependent expression of APEX2 in the transgenic line Rorb-IRES2-Cre in (A) dendritic spines, and (B) boutons. 